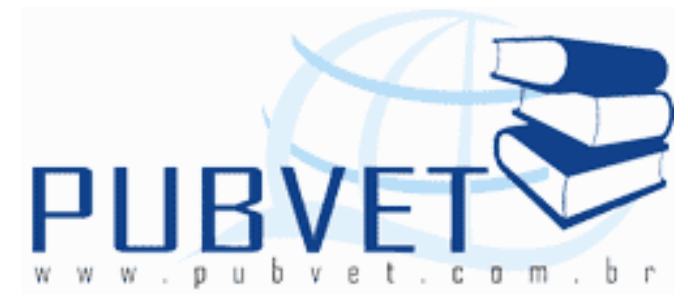

PUBVET, Publicações em Medicina Veterinária e Zootecnia.

\title{
Resposta de Cassia rotundifolia à inoculação de micorrizas arbusculares
}

Newton de Lucena Costa ${ }^{1}$, Tiago Simey Paulino ${ }^{2}$, Valdinei Tadeu Paulino ${ }^{3}$, Lucia Elenícia da Silva Nascimento ${ }^{4}$, Thiago da Silva Nascimento ${ }^{5}$, João Avelar Magalhães ${ }^{6}$

${ }^{1}$ Eng. Agr., M.Sc., Embrapa Roraima, Boa Vista, Roraima.

2 Eng. Agr., B.Sc., Sementes Matsuda e Nutrição Animal, Álvares Machado, São Paulo.

${ }^{3}$ Eng. Agr., D.Sc., APTA, IZ, Nova Odessa, São Paulo.

${ }^{4}$ Estudante de Pós-Graduação em Gestão Ambiental e Ecoturismo, Faculdade Montenegro, Parnaíba, Píauí.

${ }^{5}$ Biólogo, B.Sc., Graduado pela UFPI, Parnaíba, Píauí

${ }^{6}$ Méd. Vet., D.Sc., Embrapa Meio Norte, Parnaíba, Píauí.

\section{Resumo}

Avaliou-se o efeito da inoculação de micorrizas arbusculares (MA) - Glomus fasciculatum, G. macrocarpum, G. etunicatum, Acaulospora muricata e Scutellospora heterogama, sobre o crescimento e composição química de Cassia rotundifolia CIAT-7792. Entre os fungos avaliados, os mais eficientes na produção de matéria seca (MS) foram A. muricata e S. heterogama, os quais proporcionaram incrementos de 162 e 142\%, respectivamente, em relação ao tratamento sem inoculação. As taxas de colonização radicular foram afetadas 
pelas diferentes espécies de MA e os maiores valores registrados com a inoculação de $S$. heterogama e $G$. fasciculatum. Os maiores teores de N foram obtidos com a inoculação de $G$. etunicatum, enquanto que plantas micorrizadas por $S$. heterogama e $A$. muricata apresentaram as maiores quantidades absorvidas de $\mathrm{N}$. Para o $\mathrm{P}$, os maiores teores e quantidades absorvidas foram verificados com a inoculação de $A$. muricata e $S$. heterogama. Os resultados obtidos evidenciam que a inoculação de MA incrementou significativamente os rendimentos de MS, teores e quantidades absorvidas de $\mathrm{N}$ e $\mathrm{P}$, sendo que os fungos mais efetivos, em termos de rendimento de matéria seca, foram $A$. muricata. e S. heterogama.

Palavras-chave: fósforo, nitrogênio.

\section{Response of Cassia rotundifolia to arbuscular mycorrhizal inoculation}

\section{Abstract}

We evaluated the effect of inoculation of arbuscular mycorrhizae (AM) Glomus fasciculatum, G. macrocarpum, G. etunicatum, Acaulospora muricata and Scutellospora heterogama on growth and chemical composition of Cassia rotundifolia CIAT-7792. Among the fungi evaluated, the most efficient in dry matter (DM) production were A. muricata and S. heterogama, which provided increments of 162 and $142 \%$, respectively, compared to treatment without inoculation. The rates of root colonization were affected by different species of $M A$. The highest values were recorded with the inoculation of $S$. heterogama and G. fasciculatum. The highest N contents were obtained by inoculation of $G$. etunicatum, while mycorrhizal plants by $S$. heterogama and $A$. muricata showed the highest amounts of absorbed $N$. For contents of $P$, the highest values and amounts absorbed were verified by inoculation of $A$. muricata and $S$. heterogama. The results show that inoculation of MA significantly increased DM yields, and absorbed amounts of $\mathrm{N}$ and $\mathrm{P}$, and the fungi most effective in terms of DM yield were $A$. muricata and $S$. heterogama.

Keywords: nitrogen, phosphorus. 


\section{Introdução}

O desenvolvimento de sistemas agrícolas mais sustentáveis sob o ponto de vista técnico, econômico, social e ambiental é uma das alternativas para a substituição da agricultura itinerante, caracterizada pelo binômio derruba e queima (MAGALHÃES et al., 2004; COSTA et al., 2006). A utilização de leguminosas arbustivas, na recuperação de solos degradados ou na melhoria daqueles de baixa fertilidade natural, tem sido uma prática usual nas regiões tropicais (LOCATELLI et al., 1991; COSTA et al., 2006), notadamente nas áreas destinadas à produção de alimentos básicos.

Por outro lado, a Cassia rotundifolia é uma leguminosa que ocorre desde o sudeste dos Estados Unidos até o norte da Argentina (CAMARGO \& MIOTTO, 2004) sendo utilizada na alimentação de bovinos, como observaram PARTRIDGE \& WRIGHT (1992) e CLEMENTS et al. (1996). Esta leguminosa destacou-se entre as mais promissoras para região norte do Brasil, em decorrência de sua boa adaptação a solos de baixa fertilidade, elevado rendimento de biomassa e altos teores de nutrientes (COSTA et al., 1998). No entanto, considerando-se que o fósforo é dos fatores mais limitantes ao crescimento das leguminosas (PASTORINI et al., 2000), a inoculação de micorrizas arbusculares (MA) é uma alternativa para aumentar a disponibilidade de fósforo e sua absorção pelas plantas.

Os efeitos positivos da micorrização sobre o crescimento de leguminosas dos gêneros Cajanus, Leucaena, Acacia, Desmodium, Pueraria, Stylosanthes e Sesbania foram relatados em diversos trabalhos (COSTA et al., 1991, COSTA e PAULINO, 1997, COSTA et al., 2006; SCHIAVO et al., 2010; COSTA et., 2012). Contudo, as respostas são condicionadas às interrelações entre características do solo, espécies de leguminosas e fungos micorrízicos. Vale ressaltar que o interesse em estudos sobre a biologia, diversidade e atividade dos microorganismos do solo está cada vez maior, principalmente com os que cumprem função na ciclagem de nutrientes e/ou produtividade dos ecossistemas, como por exemplo, as micorrizas arbusculares (ANGELINI et al., 2012). 
Este trabalho teve como objetivo avaliar o efeito da inoculação de micorrizas arbusculares sobre a produção de biomassa e composição química de C. rotundifolia CIAT-7792.

\section{Material e Métodos}

O ensaio foi conduzido em casa-de-vegetação, utilizando-se um Latossolo Amarelo, textura argilosa, com as seguintes características químicas: $\mathrm{pH}$ em água $(1: 2,5)=4,98 ; \mathrm{Al}=1,7 \mathrm{cmol} / \mathrm{dm}^{3} ; \mathrm{Ca}+\mathrm{Mg}=1,6 \mathrm{cmol} / \mathrm{dm}^{3} ; \mathrm{P}=$ $2 \mathrm{mg} / \mathrm{kg}$ e $\mathrm{K}=75 \mathrm{mg} / \mathrm{kg}$. O solo foi coletado na camada arável (0 a $20 \mathrm{~cm}$ ), destorroado e passado em peneira com abertura de $6 \mathrm{~mm}$, sendo a seguir esterilizado em autoclave a $110^{\circ} \mathrm{C}$, por uma hora com intervalos de 24 horas, durante três dias, a vapor fluente e pressão de 1,5 atm.

O delineamento experimental foi em blocos casualizados, com quatro repetições, sendo os tratamentos constituídos por cinco espécies de MA: Glomus fasciculatum, G. macrocarpum, G. etunicatum, Acaulospora muricata e Scutellospora heterogama. Cada unidade experimental constou de um vaso com capacidade para $3,0 \mathrm{~kg}$ de solo seco. A inoculação das MA foi realizada adicionando-se $15 \mathrm{~g}$ de inóculo/vaso (solo + esporos + raízes), contendo aproximadamente 400 esporos $/ 50 \mathrm{~g}$ de solo, o qual foi colocado numa camada uniforme cerca de $5 \mathrm{~cm}$ abaixo do nível de plantio. Após o desbaste, deixaramse duas plantas/vaso. O controle hídrico foi realizado diariamente através da pesagem dos vasos, mantendo-se o solo em $80 \%$ de sua capacidade de campo.

Doze semanas após o desbaste, as plantas foram cortadas rente ao solo, postas para secar em estufa à $65^{\circ} \mathrm{C}$, por 72 horas, sendo a seguir pesadas e moídas em peneira de $2,0 \mathrm{~mm}$. As taxas de colonização radicular foram avaliadas através da observação, ao microscópio, de 20 fragmentos de raízes com $2 \mathrm{~cm}$ de comprimento, clarificadas com $\mathrm{KOH}$ e tingidas por azul de tripano em lactofenol, segundo a técnica de PHILLIPS \& HAYMAN (1970). 


\section{Resultados e Discussão}

A análise estatística revelou significância $(P<0,05)$ para o efeito da micorrização sobre os rendimentos de matéria seca (MS). Entre os fungos avaliados, os mais eficientes foram Acaulospora muricata e Scutellospora heterogama, os quais proporcionaram incrementos de 162 e 142\%, respectivamente, em relação ao tratamento sem inoculação. Já, Glomus macrocarpum foi o fungo menos efetivo, contudo apresentou um acréscimo na produção de MS de $42 \%$, comparativamente ao tratamento testemunha (Figura 1). Da mesma forma, PAULINO et al. (1992) constataram diferenças significativas na efetividade de quatro espécies de MA no rendimento de forragem de Centrosema brasilianum, sendo os maiores valores obtidos com a inoculação de $A$. muricata e Gigaspora margarita. COSTA et al. (1991) com Leucaena leucocephala, observaram maior efetividade de $A$. muricata e $S$. heterogama, comparativamente a G. fasciculatum, G. macrocarpum e $G$. margarita. Segundo KRUCKELMANN (1975), as plantas apresentam grande variabilidade na resposta à inoculação de $M A$, a qual parece ser controlada geneticamente, através de variações fisiológicas dos endófitos e dos mecanismos de infecção, podendo ocorrer especificidade até mesmo ao nível de variedades e/ou cultivares.

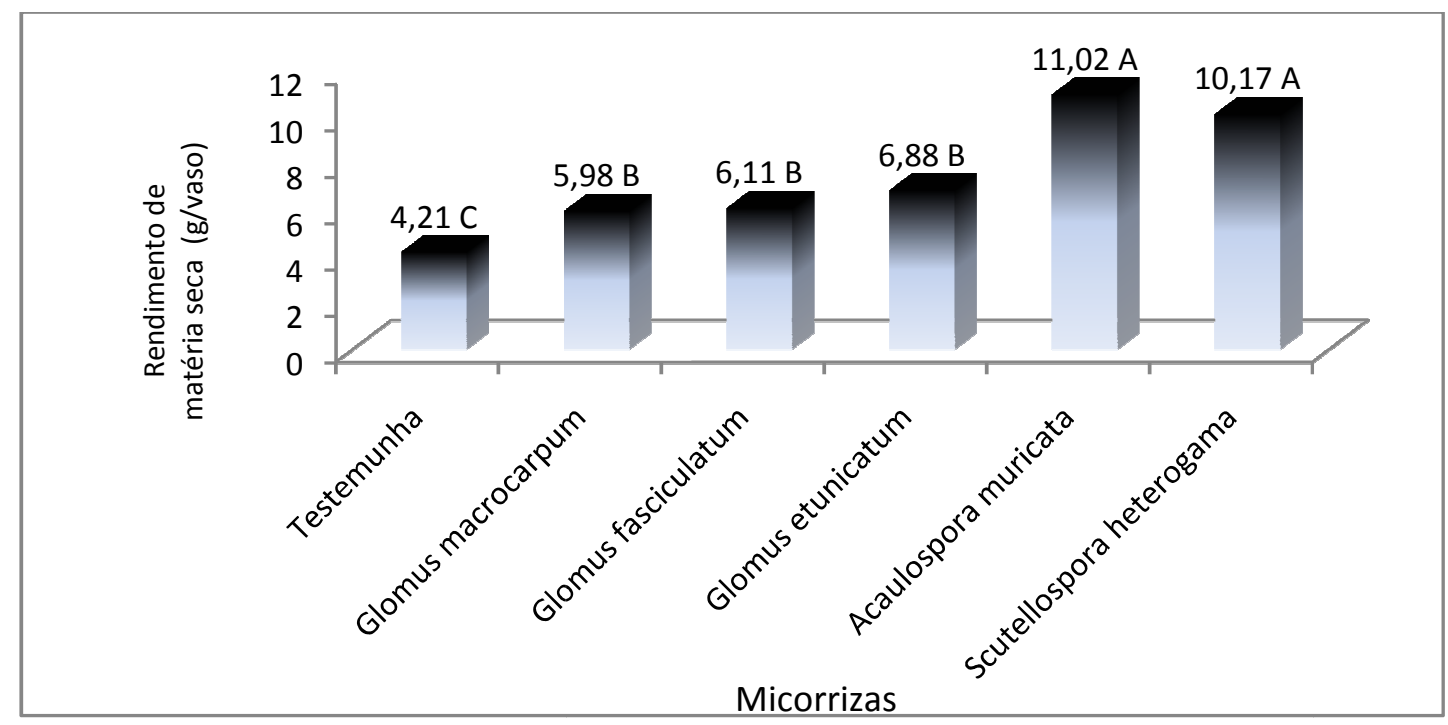

Figura 1. Rendimento de matéria seca (MS) de Cassia rotundifolia em função da inoculação de micorrizas arbusculares. 
As taxas de colonização radicular foram afetadas $(P<0,05)$ pelas diferentes espécies de MA. Os maiores valores foram registrados com a inoculação de S. heterogama e G. fasciculatum (Figura 2). COSTA et al. (2012) também reportaram que as espécies $S$. heterogama e $G$. fasciculatum apresentaram maiores taxas de colonização radicular em Sesbania sesban.

Conforme MENGE et al. (1978), o mecanismo que regula a relação entre infecção das raízes por MA não é ainda bem conhecido, porém deve estar associado ao nível crítico interno de $\mathrm{P}$ da planta hospedeira. Neste trabalho observou-se esta tendência, pois maiores taxas de colonização radicular não refletiram, necessariamente, em maiores teores de $\mathrm{P}$ nos tecidos das plantas.

MIRANDA et al. (1989) demonstraram que existe um balanço entre o fósforo do solo e do tecido que controla esta relação simbiótica. $O$ efeito do $P$ do solo seria provavelmente mais evidente na fase inicial de colonização radicular, quando o fungo está se desenvolvendo no solo, seja na germinação dos esporos ou no crescimento micelial anterior à penetração na raiz. De acordo com GREEN et al. (1976), geralmente as espécies dos gêneros Gigaspora, Scutellospora e Acaulospora ocorrem em uma faixa maior de $\mathrm{pH}$, apresentando melhor adaptação e maior efetividade em solos ácidos que as de Glomus.

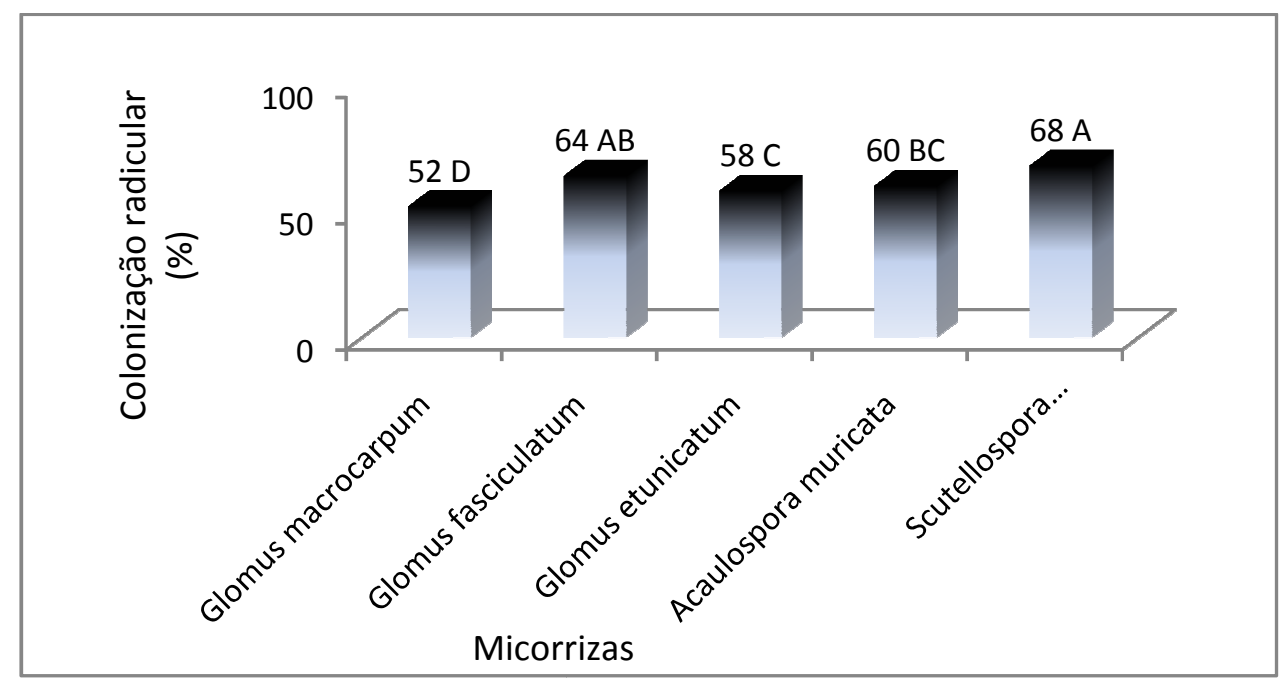

Figura 2. Colonização radicular de Cassia rotundifolia, em função da inoculação de micorrizas arbusculares. 
Os maiores teores de $\mathrm{N}$ foram obtidos com a inoculação de $\mathrm{G}$. etunicatum e com o tratamento testemunha, enquanto que plantas micorrizadas por $S$. heterogama e $A$. muricata apresentaram as maiores quantidades absorvidas de N. Para $\circ \mathrm{P}$, as maiores concentrações e quantidades absorvidas foram verificadas com a inoculação de $A$. muricata e $S$. heterogama (Tabela 1). O estímulo no crescimento da planta atribuído aos fungos micorrízicos estão fortemente correlacionados com o maior acúmulo de nutrientes, especialmente o $\mathrm{P}$ (MUNNS \& MOSSE, 1980). As plantas micorrizadas são favorecidas, pois as hifas do fungo podem beneficiar-se do $P$ distante da rizosfera que permanece inacessível às plantas não micorrizadas. Ademais, que no solo o $\mathrm{P}$ pode sofrer ataque de fosfatases fúngicas e ser solubilizado e translocado para a planta. O fósforo apresenta pouca mobilidade no solo e chega até as raízes pelo processo de difusão. Os fungos micorrízicos arbusculares (FMAs) facilitam este processo transportando o nutriente pelas suas hifas asseptadas, permitindo o crescimento e desenvolvimento das plantas em solos relativamente pobres e, ou deficientes (SANTOS, 2008).

Tabela 1. Teores e quantidades de nitrogênio $(N)$ e fósforo (P) absorvidas em Cassia rotundifolia, em função da micorrização.

\begin{tabular}{lcccc}
\hline \multicolumn{1}{c}{ Tratamentos } & $\mathrm{N}(\mathrm{g} / \mathrm{kg})$ & $\mathrm{N}(\mathrm{g} / \mathrm{vaso})$ & $\mathrm{P}(\mathrm{g} / \mathrm{kg})$ & $\mathrm{P}(\mathrm{g} / \mathrm{vaso})$ \\
\hline Testemunha & $30,22 \mathrm{a}$ & $12,72 \mathrm{~d}$ & $1,55 \mathrm{~d}$ & $0,62 \mathrm{~d}$ \\
Glomus macrocarpum & $29,12 \mathrm{~b}$ & $17,41 \mathrm{c}$ & $1,66 \mathrm{c}$ & $0,99 \mathrm{c}$ \\
Glomus fasciculatum & $26,37 \mathrm{c}$ & $16,11 \mathrm{c}$ & $1,63 \mathrm{c}$ & $1,00 \mathrm{bc}$ \\
Glomus etunicatum & $30,11 \mathrm{a}$ & $20,71 \mathrm{~b}$ & $1,64 \mathrm{c}$ & $1,13 \mathrm{~b}$ \\
Acaulospora muricata & $28,26 \mathrm{~b}$ & $31,14 \mathrm{a}$ & $1,79 \mathrm{a}$ & $1,97 \mathrm{a}$ \\
Scutellospora heterogama & $29,03 \mathrm{~b}$ & $29,52 \mathrm{a}$ & $1,73 \mathrm{~b}$ & $1,76 \mathrm{a}$ \\
\hline
\end{tabular}

Médias seguidas de mesma letra não diferem entre si $(P>0,05)$ pelo teste de Tukey 
COSTA, N.L. et al. Resposta de Cassia rotundifolia à inoculação de micorrizas arbusculares. PUBVET, Londrina, V. 7, N. 5, Ed. 228, Art. 1512, Março, 2013.

\section{Conclusões}

A inoculação de MA incrementou significativamente os rendimentos de MS, teores e quantidades absorvidas de nitrogênio e fósforo da Cassia rotundifolia, sendo que os fungos mais efetivos, em termos de rendimento de MS, foram A. muricata e S. heterogama.

\section{Referências Bibliográficas}

ANGELINI, G.A.R.; LOSS, A.; PEREIRA, M.G.; TORRES, J.L.R.; SAGGIN JÚNIOR, O.J. Colonização micorrízica, densidade de esporos e diversidade de fungos micorrízicos arbusculares em solo de cerrado sob plantio direto e convencional. Semina. Ciências Agrárias, v.33, p.115-130, 2012.

CAMARGO, R.A.; MIOTTO, T.S. O gênero Chamaecrista Moench (LeguminosaeCaesalpinioideae) no Rio Grande do Sul. Iheringia, Série Botânica, v.59, n.2, p.131-148, 2004.

CLEMENTS, R.J.; JONES, R.M.; VALDES, L.R.; BUNCH, G.A. Selection of Chamaecrista rotundifolia by cattle. Tropical Grasslands, v.30, n.4, p.389-394, 1996.

COSTA, N. de L.; LEÔNIDAS, F.C.; TOWNSEND, C.R.; MAGALHÃES, J.A.; VIEIRA, A.H. Avaliação de leguminosas arbóreas e arbustivas de múltiplo uso em Rondônia. Porto Velho: Embrapa Rondônia, 11p. 1998. (Boletim de Pesquisa, 27).

COSTA, N. de L.; MAGALHÃES, J.A.; PAULINO, V.T.; PEREIRA, R.G.A. Utilização de sistemas silvipastoris na Amazônia Ocidental Brasileira. Revista Electrónica de Veterinaria, v.2, p.1$16,2006$.

COSTA, N. de L.; PAULINO, V.T. Growth response of Acacia angustissima to vesiculararbuscular mycorrhizal inoculation. Forest, Farm and Community Tree Research Reports, v.4, p.51-53, 1997.

COSTA, N. de L.; PAULINO, V.T.; LUCENA, M.A.C.; SCHUNKE, R.; LOPES, R.B.; MAGALHÃES, J.A.; NASCIMENTO, L.E.S. Resposta de Pueraria phaseoloides (Benth.) à adubação fosfatada em presença ou não de fungo micorrízico. Pubvet, v.6, Art\#1315, 2012.

COSTA, N. de L.; PAULINO, V.T.; VEASEY, E.A.; LEÔNIDAS, F.C. Growth responses of leucaena to vesicular-arbuscular mycorrhizal inoculation. Leucaena Research Reports, v.12, p.12-13, 1991.

GREEN, N.E.; GRAHAM, S.S.; SCHENCK, N.C. The influence of $\mathrm{pH}$ on the germination of vesiculararbuscular mycorrhiza spores. Mycologia, v.68, p.929-934, 1976.

KRUCKELMANN, H.W. Effects of fertilizers, soils, soil tillage and plant species on the frequency of Endogone chlamydospores and mycorrhizal infection in arable soil. In: SANDERS, F.E.; MOSSE, B.; TINKER, P.B. (Eds.) Endomycorrhizas. London: Academic Press, 1975. p.511526.

LOCATELLI, M.; PALM, C.A.; SMYTH, T.J.; RICCI, M.S.F. Seleção de leguminosas para cultivo alley-cropping sob condições de Latossolo Amarelo em Porto Velho, Rondônia, Brasil. Porto Velho: EMBRAPA-CPAF Rondônia, 1991. 7p. (Pesquisa em Andamento, 125).

MAGALHÃES, J.A.; COSTA, N. de L.; PEREIRA, R.G.A.; TOWNSEND, C.R.; BIANCHETTI, A. Sistemas silvipastoris: alternativa para Amazônia. Revista Bahia Agrícola, v.6, n.3, p.52-54, 2004. 
MENGE, J.A.; STEIRLE, D.; BAGYARAJ, D.J.; JONHSON, E.L.V.; LEONARD, R.T. Phosphorus concentrations in plants responsible for inhibition of mycorrhizal infections. New Phytologist, v.80, p.75-80, 1978.

MIRANDA, J.C.C.; HARRIS, P.J.; WILD, A. Effects of soil and plant phosphorus concentrations on vesicular-arbuscular mycorrhiza in sorghum plants. New Phytologist, v.12, p.405-410, 1989.

MUNNS, D.N.; MOSSE, B. Mineral nutrition of legume crops. In: SUMMERFIELD, R.J.; BUNTING, A.H. (Eds.). Advances in legume science. University of Reading Press, Reading, United Kingdon, 1980. p.115-125.

PARTRIDGE, I.J.; WRIGHT, J.W. The value of round-leafed cassia (Cassia rotundifolia cV. Wynn) in native pasture grazed with steers in south-east Queensland. Tropical Grasslands, v.26, n.4, p.253-268, 1992.

PASTORINI, L.H.; BACARIN, M.A.; LOPES, N.F.; LIMA, M. das G. de S. Crescimento inicial do feijoeiro submetido a diferentes doses de fósforo em solução nutritiva. Revista Ceres, v.47, n.270, p.219-228, 2000.

PAULINO, V.T.; COSTA, N. de L.; RODRIGUES, A.N.A.; CHAGAS, F. das. Eficiência de fungos micorrízicos vesículo-arbusculares e da adubação fosfatada em Centrosema brasilianum (L.)Benth. Pasturas Tropicales, v.14, n.3, p.14-17, 1992.

PHILLIPS, J.M.; HAYMAN, D.S. Improved procedure for clearing roots and staining parasitic and vesicular-arbuscular mycorrhizal fungi for rapid assessement for infection. Transactions of the British Mycological Society, v.55, p.158-161, 1970.

SANTOS, L.C.S. Crescimento inicial de leguminosas forrageiras tropicais inoculadas com bactérias fixadoras de nitrogênio. 2008. 52 f. Dissertação (Mestrado em Produção Vegetal)- Universidade Estadual de Santa Cruz, UESC, Ilhéus, 2008.

SCHIAVO, J.A.; MARTINS, M.A.; RODRIGUES, L.A. Crescimento de mudas de Acacia mangium, Sesbania virgata e Eucalyptus camaldulensis, inoculadas com fungos micorrízicos, em casa-devegetação e em cava-de-extração de argila. Acta Scientiarum. Agronomy, v.32, n.1, p.171$178,2010$. 